\title{
Changes of serum advanced glycation end products (AGEs), matrix metalloprotein-2 (MMP-2), and urinary microalbuminuria (mALB) in diabetic nephropathy and their predictive value for heart
} failure

\author{
Xieyi Sun ${ }^{1,2}$, Hua Gan ${ }^{3}$, Yunfeng Xia ${ }^{3}$ \\ ${ }^{1}$ Chongqing Medical University, Chongqing, China; ${ }^{2}$ Department of Nephrology, the People's Hospital of Rongchang District, Chongqing, China; \\ ${ }^{3}$ Department of Nephrology, the First Affiliated Hospital of Chongqing Medical University, Chongqing, China \\ Contributions: (I) Conception and design: X Sun; (II) Administrative support: H Gan; (III) Provision of study materials or patients: X Sun, Y Xia; (IV) \\ Collection and assembly of data: All authors; (V) Data analysis and interpretation: H Gan, Y Xia; (VI) Manuscript writing: All authors; (VII) Final \\ approval of manuscript: All authors. \\ Correspondence to: Hua Gan; Yunfeng Xia. Department of Nephrology, the First Affiliated Hospital of Choingqing Medical University. Youyi Road, \\ Yuanjiagang, Yuzhong District, Chongqing 400016, China. Email: ganhua0000@163.com; haohua11234@sina.com.
}

Background: Diabetic nephropathy is a common complication in diabetic patients, with a high rate of disability and mortality. This study aims to explore the changes in serum advanced glycation end products (AGEs), matrix metalloprotein-2 (MMP-2), and urinary microalbuminuria (mALB) in diabetic nephropathy and their predictive value for heart failure.

Methods: The 134 patients with diabetic nephropathy treated in our hospital from January 2014 to December 2017 were enrolled and divided into two groups resulting in 64 cases in an observation group with heart failure, and 70 cases without heart failure in a control group. In addition, 80 patients with simple diabetes who were treated during the same period were selected as the simple diabetes group. Levels of AGEs, MMP-2, and mALB between the groups were compared, risk factors affecting diabetic nephropathy patients with heart failure were analyzed, and an ROC curve was drawn to evaluate the predictive value of AGEs, MMP-2, and mALB for heart failure

Results: The levels of AGEs and mALB in the diabetic nephropathy group were significantly higher than those in the simple diabetes group, and the levels of MMP-2 were significantly lower than those in the simple diabetes group $(\mathrm{P}<0.05)$. The levels of AGEs and mALB in the observation group were significantly higher than those in the control group, and the levels of MMP-2 were significantly lower than that in the control group $(\mathrm{P}<0.05)$. Smoking history hypertension history, blood creatinine (abnormal increase), blood uric acid (abnormal increase), AGEs (abnormal increase), MMP-2 (abnormal decrease), and mALB (abnormal increase) were independent risk factors affecting diabetic nephropathy patients with heart failure. The area under the ROC curve of AGEs, MMP-2, mALB, and their combined detection were: 0.821, 0.909, 0.897, and 0.991 , respectively, showing the area under the curve of combined detection to be the largest.

Conclusions: AGEs, MMP-2, and MALB have high predictive value for heart failure in patients with diabetic nephropathy. Their sensitivity and specificity are high, indicating they may hold considerable clinical value.

Keywords: Advanced glycation end products (AGEs); matrix metalloprotein-2 (MMP-2); microalbuminuria (mALB); diabetic nephropathy; heart failure

Submitted Dec 17, 2020. Accepted for publication Feb 10, 2021.

doi: $10.21037 /$ tau-21-35

View this article at: http://dx.doi.org/10.21037/tau-21-35 


\section{Introduction}

Diabetic nephropathy is one of the common complications of diabetes mellitus, which is induced by the metabolic abnormalities and the related damage to renal microvessels that caused by hyperglycemia. With the progression of the disease, diabetic nephropathy may develop to urinary toxicity with a high disability and mortality rate (1). Most diabetic patients are complicated with coronary heart disease, cardiomyopathy and other cardiovascular diseases. After kidney damage, cardiovascular disease and other factors lead to the progression of the disease, and the patients with excessive body fluid load may also be complicated with heart failure. Related literature has reported that the incidence of heart failure in diabetic patients is about $22 \%$ (2). Diabetic nephropathy combined with heart failure generally has a long course and progresses slowly. Before the symptoms of proteinuria and heart failure appear in patients, it is easy to be ignored. Therefore it is of great significance to timely diagnose patients and determine the treatment plan at the early stage to improve the prognosis of patients. Previous studies have shown that indexes such as urine protein, creatinine, and urea nitrogen are poor diagnostic indicators of the disease (3). Therefore, there is an urgent clinical need to find new and effective biomarkers to improve the efficacy of early diagnosis of patients with diabetic nephropathy.

Some scholars suggest that urine microalbuminuria (mALB) is overexpressed in patients with diabetic nephropathy and heart failure and may serve as useful biomarkers in disease detection and as prognostic indicators (4). Serum advanced glycation end products (AGEs) is one of the indicators to detect a variety of chronic diseases. High level of AGEs is an important pathological basis for inducing diabetic nephropathy (5). Other studies have also reported that the overexpression of matrix metalloproteinases (MMPs) imbalances the synthesis and degradation of the glomerular extracellular matrix, leading to its accumulation which in turn leads to renal fibrosis in patients with diabetic nephropathy. Matrix metalloprotein-2 (MMP-2) may play an important role in the occurrence and development of diabetic nephropathy (6). However, the sensitivity and specificity of combined detection of mALB, AGEs and MMPs in patients with diabetic nephropathy complicated with heart failure has not been clearly determined. This study aims to explore the changes of mALB, AGEs, and MMP-2 in patients with diabetic nephropathy, assess the predictive value of these three indicators in diabetic nephropathy patients with heart failure, and provide a theoretical basis for improving clinical diagnostic efficacy.

We present the following article in accordance with the STARD reporting checklist (available at http://dx.doi. org/10.21037/tau-21-35).

\section{Methods}

\section{General information}

The clinical data of diabetic nephropathy patients treated in our inpatient department from January 2014 to December 2017 were collected and sorted. The following inclusion criteria were used: (I) patients with diabetes symptoms such as polyuria, polydipsia, weight loss caused by unknown causes, blood glucose $\geq 7.0 \mathrm{mmol}$ for 3 consecutive days, and diagnosed with diabetes; (II) patients met the diagnostic criteria for nephropathy according to the Chinese medical association clinical diagnostic criteria for nephropathy (7); (III) patients met the diagnostic criteria in "China Heart Failure Diagnosis and Treatment Guidelines 2014" formulated by Chinese Society of Cardiology of Chinese Medical (8); (IV) patients aged under 80 years old; The exclusion criteria were as follows: (I) patients who had taken drugs for the control of blood glucose and serum inflammatory factors before being included in the study; (II) patients with concomitant malignant tumors or severe diseases of the vital organs; (III) patients who were likely to have poor treatment compliance; (IV) women during pregnancy and lactation; and $(\mathrm{V})$ patients with heart failure caused by hyperthyroidism, rheumatic immune system diseases, and other factors.

A total of 134 patients with diabetic nephropathy met the above criteria, and these were then divided into two groups according to the presence or absence of heart failure; an observation group (64 cases, with heart failure) and a control group (70 cases, without heart failure). All patients were in accordance with the signs of cardiac function classification where their left ventricular ejection fraction was less than $50 \%$ and left ventricular end-diastolic period was over $55 \mathrm{~mm}$ (9). There were 34 males and 30 females in the observation group, aged 43-75 years old, with an average age of $(55.41 \pm 3.31)$ years, and an average disease course of $(7.01 \pm 1.33)$ years. In the control group, there were 36 males and 34 females, aged $45-76$ years old, with an average age of $(55.48 \pm 3.35)$ years, and an average disease course of $(7.05 \pm 1.37)$ years. In addition, 80 patients with simple 
Table 1 Comparison of AGEs, MMP-2, and mALB levels in simple diabetes and diabetic nephropathy $(\bar{x} \pm s)$

\begin{tabular}{lcccc}
\hline Group & Case & AGEs $(\mathrm{ng} / \mathrm{mL})$ & MMP-2 $(\mathrm{pg} / \mathrm{mL})$ & $\mathrm{mALB}(\mathrm{mg} / \mathrm{L})$ \\
\hline Simple diabetes & 80 & $1.12 \pm 0.75$ & $512.35 \pm 49.77$ & $13.65 \pm 1.72$ \\
Diabetic nephropathy & 134 & $2.75 \pm 1.20$ & $450.15 \pm 42.65$ & $38.65 \pm 12.54$ \\
$T$ & - & 10.935 & 9.690 & 17.716 \\
$P$ & - & 0.001 & 0.001 & 0.001 \\
\hline
\end{tabular}

AGEs, advanced glycation end products; MMP-2, matrix metalloprotein-2; mALB, microalbuminuria.

diabetes who were treated in the same hospital during the same period were selected as the simple diabetes group which was composed of 32 males and 28 females, aged between $47-78$ years, with an average age of $(55.57 \pm 3.39)$ years old, and an average disease course of $(6.88 \pm 1.29)$ years. There was no statistically significant difference in baseline data of patients in each group $(\mathrm{P}>0.05)$. This study was approved by the People's Hospital of Rongchang District [No. 2021-(1)]. All procedures performed in this study involving human participants were in accordance with the Declaration of Helsinki (as revised in 2013). Informed consent was taken from all the patients.

\section{Detection method of AGEs and MMP-2 levels}

A $3 \mathrm{ml}$ sample of venous blood was drawn from all subjects on an empty stomach on the morning of each evaluation. This was centrifuged for 10 minutes to separate serum and plasma and the serum samples were stored at $-20{ }^{\circ} \mathrm{C}$ for further testing. The levels of AGEs and MMP-2 were detected using the Enzyme-linked immunosorbent assay (ELISA) purchased from Beijing Zhongshan Jinqiao Biotechnology (Beijing, China).

\section{Detection method of mALB level}

Morning urine $(5 \mathrm{~mL})$ was collected from the study subjects, followed by centrifuging for $10 \mathrm{~min}$. Then mALB level in the supernatant was detected by the fully automatic specific protein analyzer (A15) and its supporting kits (BioSystems, Spain). The detection process was carried out in strict accordance with the kit instructions.

\section{Observation indicators}

Firstly, the levels of AGEs, MMP-2, and mALB were compared between patients with simple diabetes and diabetic nephropathy, then between the observation group and the control group. Multiple logistic regression was performed to analyze the related risk factors of diabetic nephropathy patients. In addition, an ROC curve was drawn to analyze the predictive value of AGEs, MMP-2, and mALB levels.

\section{Statistical analysis}

Statistical analysis was performed using SPSS22.0 software (IBM, New York, USA). The count data was expressed as $\mathrm{n}(\%)$, and analyzed using $t$ test, and measurement data were described using mean \pm standard deviation $(\bar{x} \pm s)$ and analyzed by chi-squared $\left(\chi^{2}\right)$ test. Multiple logistic regression analysis was used to analyze the risk factors influencing patients with diabetic nephropathy with heart failure. The ROC curve was drawn and indicators such as the area under the ROC curve were calculated to analyze the predictive value of AGEs, MMP-2, and mALB levels. Results with $\mathrm{P}<0.05$ indicated significant difference.

\section{Results}

\section{Comparison of AGEs, MMP-2, and mALB levels between simple diabetes and diabetic nephropatby}

The results showed that levels of AGEs and mALB in the diabetic nephropathy group were significantly higher than those in the simple diabetes group, and the level of MMP2 was significantly lower than that in the simple diabetes group $(\mathrm{P}<0.05$, Table 1).

\section{Comparison of AGEs, MMP-2, and $m A L B$ levels in diabetic nephropathy patients with or without heart failure}

The levels of AGEs and mALB in the observation group (diabetic nephropathy patients with heart failure) were significantly higher than those in the control group (diabetic 
Table 2 Comparison of AGEs, MMP-2, and mALB levels in patients with or without heart failure $(\bar{x} \pm \mathrm{s})$

\begin{tabular}{lcccc}
\hline Group & Case & AGEs $(\mathrm{ng} / \mathrm{mL})$ & MMP-2 $(\mathrm{pg} / \mathrm{mL})$ & $\mathrm{mALB}(\mathrm{mg} / \mathrm{L})$ \\
\hline Observation group & 64 & $3.35 \pm 1.39$ & $418.32 \pm 30.71$ & $37.65 \pm 10.65$ \\
Control group & 70 & $2.88 \pm 1.27$ & $443.18 \pm 35.21$ & $20.14 \pm 8.14$ \\
$t$ & - & 2.045 & 4.338 & 10.746 \\
$P$ & - & 0.043 & 0.001 & 0.001 \\
\hline
\end{tabular}

AGEs, advanced glycation end products; MMP-2, matrix metalloprotein-2; mALB, microalbuminuria.

Table 3 Analysis of single and multiple factors affecting diabetic nephropathy patients with heart failure

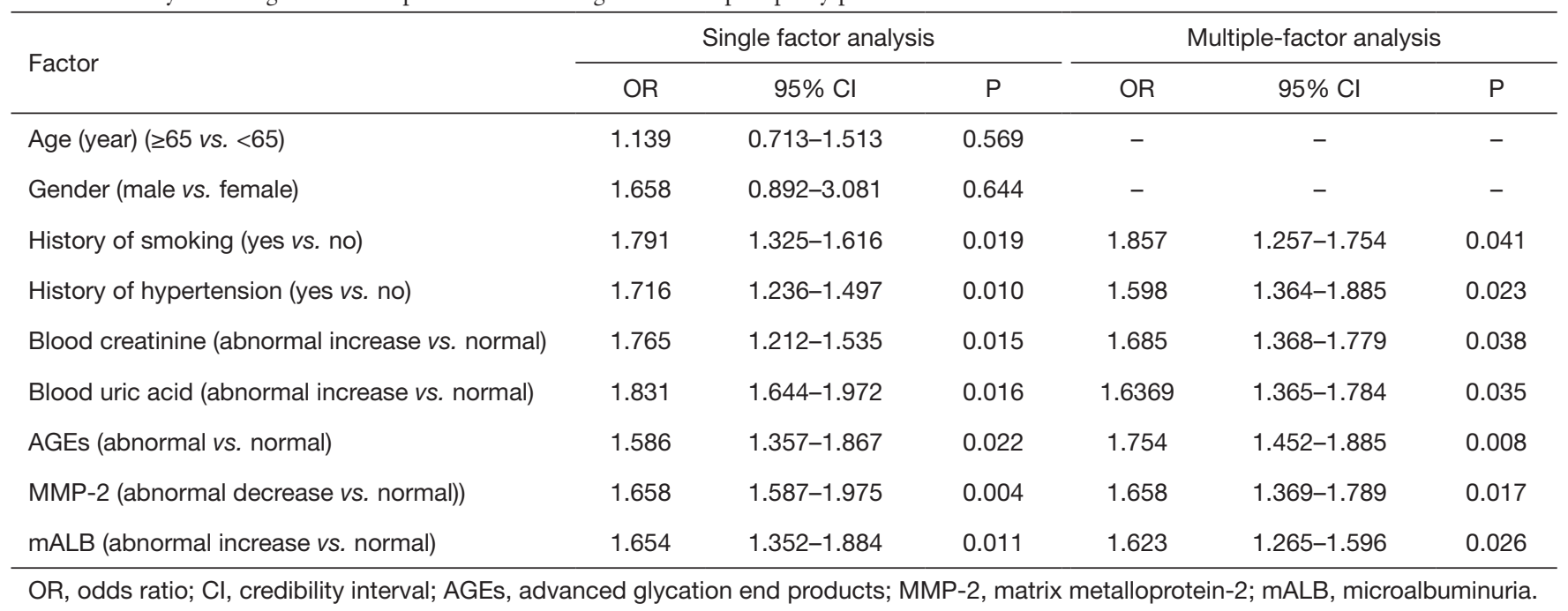

nephropathy patients without heart failure), and the level of MMP-2 in the observation group were significantly lower than that in the control group $(\mathrm{P}<0.05$, Table 2$)$.

\section{Analysis of single and multiple factors affecting diabetic nephropathy patients with beart failure}

Factors such as a smoking history, hypertension history, blood creatinine (abnormal increase), blood uric acid (abnormal increase), AGEs (abnormal increase), MMP-2 (abnormal decrease), and mALB (abnormal increase) were independent risk factors affecting patients with diabetic nephropathy and heart failure (Table 3).

\section{The predictive value of AGEs, MMP-2, and $m A L B$ levels in diabetic nephropathy patients with heart failure}

The area under the ROC curve of AGEs, MMP-2, mALB, and the three combined detection were $0.821,0.909,0.897$, and 0.991, respectively, indicating that the area under the curve was the largest in the combined detection of the three indicators (Table 4 and Figure 1).

\section{Discussion}

Diabetic nephropathy is usually caused by metabolic abnormalities and damage to small blood vessels in the kidney, and is a common complication in diabetics (10). Many patients suffer from high blood sugar, and when not effectively controlled this can lead to vascular disease and kidney damage. This can result in a decrease in left ventricular ejection volume of the heart, accelerating vasoconstriction, reducing blood flow in the kidneys, inducing water and sodium retention, and eventuate in heart failure. The combined effect of diabetes nephropathy and heart failure imposes significant detrimental effects on the lives of those affected and the identification of meaningful biomarkers to assist in the early diagnosis and treatment of patients is urgently required.

AGEs are a class of molecular glycosylation product 
Table 4 The predictive value of AGEs, MMP-2, and mALB levels in patients with diabetic nephropathy complicated with heart failure

\begin{tabular}{lccccc}
\hline Predictive indicator & Optimal threshold & Sensitivity (\%) & Specificity (\%) & AUC & 95\% Cl \\
\hline AGEs & 26.41 & 0.894 & 0.924 & 0.821 & $0.710-0.933$ \\
MMP-2 & 26.30 & 0.876 & 0.883 & 0.909 & $0.831-0.987$ \\
mALB & 26.24 & 0.914 & 0.897 & $0.808-0.987$ \\
Combination of the three & 26.81 & 0.934 & 0.948 & 0.991 & $0.000-1.000$ \\
Predictive indicator model & 1.711 & 0.875 & 0.889 & $0.7548-0.995$ \\
\hline
\end{tabular}

AGEs, advanced glycation end products; MMP-2, matrix metalloprotein-2; mALB, microalbuminuria; AUC, area under the curve; Cl, credibility interval.

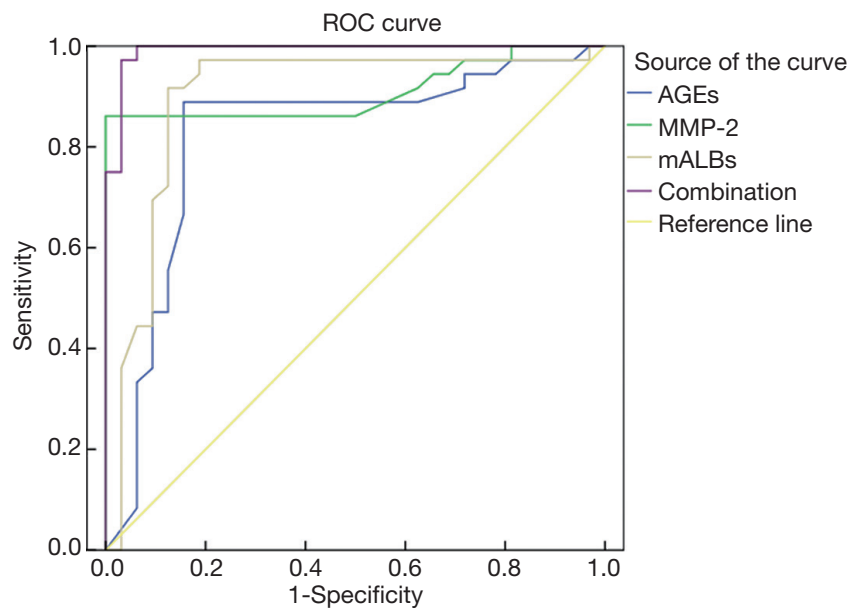

Figure 1 Predictive value of AGEs, MMP-2, and mALB levels in patients with diabetic nephropathy combined with heart failure. AGEs, advanced glycation end products; MMP-2, matrix metalloprotein-2; mALB, microalbuminuria.

which are widely used as indicators for understanding of the progression of chronic diseases (11). Previous studies have shown $(10,12)$ that the overexpression of AGEs can promote the occurrence and progression of diabetic nephropathy and damage the kidneys, by aggravating water and sodium retention leading to heart failure. Scholars such as Yuan CM have reported that AGEs are highly expressed in coronary heart disease and other cardiovascular diseases (13). Urine $\mathrm{mALB}$ is a sensitive and reliable diagnostic indicator for the early detection of kidney damage in diabetic patients (14) and has been used to monitor the occurrence of diabetic nephropathy. This study found that levels of serum AGEs and $\mathrm{mALB}$ in the diabetic nephropathy group were significantly higher than those in the simple diabetes group; and the serum AGEs and mALB levels in the diabetic nephropathy group with heart failure were significantly higher than those in the diabetic nephropathy group without heart failure. These results confirm the observation that levels of serum AGEs and mALB are abnormally increased in patients with heart failure and in diabetic patients with kidney damage.

As a kind of collagenase with a degrading effect, MMP2 is widely expressed in glomerular mesangial cells and can maintain the dynamic balance of extracellular matrix $(15,16)$. Ma et al. (17) have pointed out that changes in MMP-2 levels are negatively correlated with the urinary albumin excretion rate, and its concentration will increase as renal function weakens. In the present study the urine MMP-2 level in the diabetic nephropathy group with heart failure was significantly lower than that of the diabetic nephropathy group without heart failure; and the urine MMP-2 level of the diabetic nephropathy group was significantly lower than that of the simple diabetes group. The decrease of MMP2 levels in patients with diabetic nephropathy might be due to severe damage to the vascular endothelium, the increase of intercellular matrix components in the glomerular mesangial area, and the augment of urinary albumin excretion (18-20). Our results also showed that factors such as a history of smoking (yes), history of hypertension (yes), blood creatinine (abnormal increase), blood uric acid (abnormal increase), AGEs (abnormal increase), MMP-2 (abnormal decrease), and mALB (abnormally increase) were all independent risk factors affecting diabetic nephropathy patients combined with heart failure. This suggests that the occurrence of diabetic nephropathy combined with heart failure is a complex process influenced by multiple factors, including modifiable risk lifestyle risk factors which should be a focus for intervention. By drawing the ROC curve of AGEs, MMP-2, mALB, and the combined detection of the three indicators, we found that the area under the ROC curve was the largest by combined detection of the three. This suggests that the combined detection of serum AGEs, 
MMP-2, and urine mALB levels in patients with diabetic nephropathy can be used to predict the occurrence of heart failure clinically.

In summary, AGEs, MMP-2, and mALB have high predictive value for diabetic nephropathy patients with heart failure. The sensitivity and specificity of the three indicators are high, indicating their significant clinical value. However, due to the small sample size of cases and short study time in this study, the correlations in AGEs, MMP-2 and mALB needs to be further studied.

\section{Acknowledgments}

Funding: None.

\section{Footnote}

Reporting Checklist: The authors have completed the STARD reporting checklist. Available at http://dx.doi.org/10.21037/ tau-21-35

Data Sharing Statement: Available at http://dx.doi. org/10.21037/tau-21-35

Conflicts of Interest: All authors have completed the ICMJE uniform disclosure form (available at http://dx.doi. org/10.21037/tau-21-35). The authors have no conflicts of interest to declare.

Ethical Statement: The authors are accountable for all aspects of the work in ensuring that questions related to the accuracy or integrity of any part of the work are appropriately investigated and resolved. This study was approved by the People's Hospital of Rongchang District [No. 2021-(1)]. All procedures performed in this study involving human participants were in accordance with the Declaration of Helsinki (as revised in 2013). Informed consent was taken from all the patients.

Open Access Statement: This is an Open Access article distributed in accordance with the Creative Commons Attribution-NonCommercial-NoDerivs 4.0 International License (CC BY-NC-ND 4.0), which permits the noncommercial replication and distribution of the article with the strict proviso that no changes or edits are made and the original work is properly cited (including links to both the formal publication through the relevant DOI and the license). See: https://creativecommons.org/licenses/by-nc-nd/4.0/.

\section{References}

1. Wang WG, An LL, Zhang XY, et al. Effect of Low-protein Diet based on Carbohydrate Counting on Patients with Early Diabetic Nephropathy. J Med Res 2020;31:111-5.

2. Heerspink HJL, Andress DL, Bakris G, et al. Rationale and protocol of the Study Of diabetic Nephropathy with AtRasentan (SONAR) trial: A clinical trial design novel to diabetic nephropathy. Diabetes Obes Metab 2018;20:1369-76.

3. Alzahrani S, Zaitone SA, Said E, et al. Protective effect of isoliquiritigenin on experimental diabetic nephropathy in rats: Impact on Sirt-1/NFkB balance and NLRP3 expression. Int Immunopharmacol 2020;87:106813.

4. Zhang L, Zhao S, Zhu Y. Long noncoding RNA growth arrest-specific transcript 5 alleviates renal fibrosis in diabetic nephropathy by downregulating matrix metalloproteinase 9 through recruitment of enhancer of zeste homolog 2. FASEB J 2020;34:2703-14.

5. Zhang $\mathrm{S}, \mathrm{Xu} \mathrm{L}$, Liang R, et al. Baicalin suppresses renal fibrosis through microRNA-124/TLR4/NF- $\kappa B$ axis in streptozotocin-induced diabetic nephropathy mice and high glucose-treated human proximal tubule epithelial cells. J Physiol Biochem 2020;76:407-16.

6. Goetz M, Malek NP. RBP4/Lp-PLA2/Netrin-1 signaling regulation of cognitive dysfunction in diabetic nephropathy complicated with silent cerebral infarction. Exp Clin Endocrinol Diabetes 2017;125:547-53.

7. Sulaj A, Kopf S, Gröne E, et al. ALCAM a novel biomarker in patients with type 2 diabetes mellitus complicated with diabetic nephropathy. J Diabetes Complications 2017;31:1058-65.

8. Wei Y. Major highlights of the "2014 Guidelines for the Diagnosis and Treatment of Heart Failure in China". Chinese Journal for Clinicians 2015;05:17-9.

9. Li YY, Wang H, Yang XX, et al. AR C-106T gene polymorphism and diabetic nephropathy in the Eastern Asians with T2DM: A meta-analysis including 2120 subjects. Diabetes Res Clin Pract 2017;130:244-51.

10. Kitzmiller JL, Brown ER, Phillippe M, et al. Diabetic nephropathy and perinatal outcome. Am J Obstet Gynecol 1981;141:741-51.

11. Yi H, Peng R, Zhang LY, et al. LincRNA-Gm4419 knockdown ameliorates NF-kB/NLRP3 inflammasomemediated inflammation in diabetic nephropathy. Cell Death Dis 2017;8:e2583.

12. Han Q, Zhu H, Chen X, Liu Z. Non-genetic mechanisms of diabetic nephropathy. Front Med 2017;11:319-32. 
13. Yuan CM, Nee R, Ceckowski KA, et al. Diabetic nephropathy as the cause of end-stage kidney disease reported on the medical evidence form CMS2728 at a single center. Clin Kidney J 2017;10:257-62.

14. Ma ZH, Jia SL, Gao XY, et al. Effect of Hemodialysis and Peritoneal Dialysis on the Prognosis of Patients with End-stage Diabetic Nephropathy. Progress in Modern Biomedicine 2017;17:6749-51.

15. Zhou L, Huang W, Xu Y, et al. Sweet Taste Receptors Mediated ROS-NLRP3 Inflammasome Signaling Activation: Implications for Diabetic Nephropathy. J Diabetes Res 2018;2018:7078214.

16. Takada T, Masaki T, Hoshiyama A, et al. Tolvaptan alleviates excessive fluid retention of nephrotic diabetic renal failure unresponsive to furosemide. Nephrology (Carlton) 2018;23:883-6.

17. Ma TY, Zhao Y. Changes of urinary microalbumin

Cite this article as: Sun X, Gan H, Xia Y. Changes of serum advanced glycation end products (AGEs), matrix metalloprotein-2 (MMP-2), and urinary microalbuminuria (mALB) in diabetic nephropathy and their predictive value for heart failure. Transl Androl Urol 2021;10(3):1279-1285. doi: $10.21037 /$ tau-21-35 and BNP levels in patients with diabetic nephropathy complicating heart failure and their influence on recent prognosis. Int J Lab Med 2018;33:258-74.

18. Bryson JM, Baxter RC. Adrenal involvement in the diabetes-induced loss of growth hormone and prolactin receptors in the livers of female rats. Diabetologia 1986;29:106-11.

19. Weisrock F, Fritschka M, Beckmann S, et al. Reliability of peripheral arterial tonometry in patients with heart failure, diabetic nephropathy and arterial hypertension. Vasc Med 2017;22:292-300.

20. Feodoroff M, Harjutsalo V, Forsblom C, et al. Dosedependent effect of smoking on risk of coronary heart disease, heart failure and stroke in individuals with type 1 diabetes. Diabetologia 2018;61:2580-9.

(English Language Editor: B. Draper) 